\title{
A Rasch Perspective on Firm Financial Performance in the Pharmaceutical Industry
}

\author{
Thani Jambulingam, Carolin Schellhorn, and Rajneesh Sharma
}

\begin{abstract}
The metrics that are relevant for ranking firms by their financial performance may vary with conditions across different industries. For the pharmaceutical industry, we explore an approach that lets the Rasch model determine the performance metrics that are most important. Using an initial set of ratios spanning multiple dimensions of firm financial performance, we select the ratios that are compatible with the requirements of the Rasch model for this industry during 2002-2011. We identify the metrics, for which positive results were most difficult to achieve, and the firms that most frequently ranked among the top five performers. Our approach offers a new perspective or research method on the valuation of managers and their firms. Interestingly, our results suggest that the variables most relevant for a Rasch financial performance ranking of firms in this industry are not necessarily the variables that directly measure increases in investor wealth or returns.
\end{abstract}

T. Jambulingam $(\bowtie) \bullet$ C. Schellhorn $\bullet$ R. Sharma

Saint Joseph's University, Philadelphia, PA, USA

e-mail: tjambuli@sju.edu; schellho@sju.edu; rsharma@sju.edu 